\title{
Double quantum dot turnstile as an electron spin entangler
}

\author{
Xuedong $\mathrm{Hu}^{1,2}$ and S. Das Sarma ${ }^{2}$ \\ ${ }^{1}$ Department of Physics, University at Buffalo, SUNY, Buffalo, NY 14260-1500 \\ ${ }^{2}$ Condensed Matter Theory Center, Department of Physics, \\ University of Maryland, College Park, MD 20742-4111
}

(Dated: October 29, 2018)

\begin{abstract}
We study the conditions for a double quantum dot system to work as a reliable electron spin entangler, and the efficiency of a beam splitter as a detector for the resulting entangled electron pairs. In particular, we focus on the relative strengths of the tunneling matrix elements, the applied bias and gate voltage, the necessity of time-dependent input/output barriers, and the consequence of considering wavepacket states for the electrons as they leave the double dot to enter the beam splitter. We show that a double quantum dot turnstile is, in principle, an efficient electron spin entangler or entanglement filter because of the exchange coupling between the dots and the tunable input/output potential barriers, provided certain conditions are satisfied in the experimental set-up.

PACS numbers: 03.67.Lx, 03.67.-a, 73.20.Dx, 85.30.Vw,
\end{abstract}

\section{INTRODUCTION}

Experimental and theoretical studies in quantum computing and quantum information processing have shown that there exist natural resources in the quantum regime ${ }^{1}$ such as quantum superposition and entanglement that can be exploited to provide additional computing power. In particular, the study of quantum entanglement has attracted wide spread attention because of its direct relevance to quantum computation and its implications to the foundations of quantum mechanics 1.2 Many physical systems ranging from atomic and optical to solid state have been proposed as potential candidates for quantum information processing and for providing insights to their inherent quantum mechanical properties. Specifically, localized spins (electron or nuclear) trapped in solid state host materials (particularly semiconductors) have been considered as good candidates for these purposes because of their relatively isolated (from their environment) nature ${ }^{3.4 .5 .6 .7}$ In this context, the creation and the detection of electron spin entanglement become critically important tasks, and are the subject matter of interest to us in this paper.

Quantum entanglement is a manifestly non-classical property of the quantum state of a composite system (e.g. two or more particles) where the entangled composite state cannot be decomposed into a product of the individual states of local constituents, and as such the constituents are "entangled" no matter how far they are separated spatially. The classic example is the spin singlet "EPR" state of two spin- $1 / 2$ fermions, where, no matter how far apart the two particles are spatially, a measurement of the spin of one particle completely determines the quantum spin state of the other (provided, of course, the spin singlet state of the two-particle state is preserved coherently up to the measurement process, i.e. no decoherence takes place until measurement).

Entanglement leads to specific nonlocal (and nontrivially nonclassical) correlations in the measured properties of the individual constituents, which are typically expressed in terms of the celebrated Bell's inequalities. A violation of Bell's inequalities in correlation measurements of the constituents indicates the presence of entanglement in the technical sense (i.e. shows that the state of one constituent is inextricably and nonlocally quantum-mechanically entangled with the state of the other constituent no matter how far spatially apart they may be). Direct demonstration of entanglement (as manifested in the violation of Bell's inequalities) have so far been limited mostly to experiments involving photons 8.9 .10 because entangled photons are easy to produce in laboratories using the parametric down conversion processes in optical nonlinear crystals, and photons have very long coherence lengths since they are extremely weakly interacting objects. However, from a classical perspective at least (i.e. when considering light as an all pervading wave rather than a collection of quantized photons), nonlocal entanglement manifestation of light waves is not a particularly shocking situation as it would be with classically massive objects such as atoms 11 and electrons, which are classically purely particle-like, making any nonlocal classical correlation impossible.

Entanglement (in the technical Bell's inequalities or EPR sense) has never been experimentally demonstrated in any condensed matter systems. Because of strong interactions inherently present in all solid state systems, the ground state is often an interacting many-body state (e.g. the strongly correlated Laughlin state for the fractional quantum Hall system, the Bethe ansatz singlet ground state for one-dimensional spin chains, the BCS superconducting ground state) where the collective state is highly 'entangled' in the sense that it cannot be written as a mean field product state of one electron Hartree wavefunctions $\stackrel{12.13}{=}$ While such strongly coupled states (the Laughlin state in the fractional quantum Hall system being a classic example) are intrinsically entangled by definition, in general they are unsuitable for the observation of entanglement in the technical sense of EPR/Bell's inequalities, since turning off the interactions 
and spatially separating such entangled electrons so as to carry out correlation measurements are essentially impossible in the strongly interacting solid state environment. We mention, however, that perhaps more thinking and research should go into exploring the possibility of using a strongly coupled quantum many-body ground state such as the Laughlin state to experimentally demonstrate entanglement correlations.

Experimental demonstration of solid state entanglement should entail the following minimal steps as a matter of principle: (1) Two (or more) particles (or sub-systems) interact to form an entangled pair; (2) this entangled pair

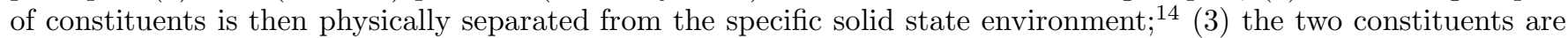
spatially separated and their interaction turned off while the entangled two-particle state is preserved,14.15 $(4)$ when the two constituents are sufficiently spatially separated, some suitable properties of each are measured in a correlated manner; (5) a study of correlation between these local measurements on each constituent, provided they violate Bell type inequalities, establishes entanglement between the constituents. We emphasize that the hardest steps in the experimental observation of solid state entanglement are the steps two and three above because it is extremely hard to spatially separate electrons in solids in a controlled manner without causing decoherence. The problem in observing solid state entanglement is really too much (and not too little) entanglement - electrons in solids are intrinsically coupled or entangled with each other (and with the environment) and extracting a pair of constituents and spatially separating them in order to observe entangled correlations within the pair is very difficult, if not impossible.

A possibly suitable method to observe solid state electronic entanglement is to use transport or tunneling techniques, which naturally allow flow or movement of particles enabling spatial separation, the essential ingredient for observing entangled correlations. For example, one could extract a Cooper pair from a superconductor, which is in an entangled (delocalized) singlet state in general. Then the two electrons in this Cooper pair have to be spatially separated somehow (using coherent tunneling or transport through various leads) in a ballistic manner (i.e. without any decoherence whatsoever) so that correlation measurements on each electron of the pair can be carried out locally on each system without any interaction with its distant partner. Clearly, decoherence will be a severe problem in such experiments as each electron of the singlet pair will necessarily interact strongly with the environment (i.e. all "other" electrons in the system and in the leads, phonons, magnetic and other impurities). In spite of all these problems, many approaches for creating/detecting entanglement in solid state systems (involving, for example, delocalized Cooper pairs 16.17.18.19.20.21, localized quantum dot or quantum wire electron singlets 22.23 .24 , beam splitters 25.26 .27 .28 .29 , etc) have been theoretically proposed in the literature to study spin entanglement properties in a solid state environment, and in this paper we study in some depth a proposal in which electron spin entanglement is generated in a coupled double quantum dot and detected through a beam splitter, where measurements on the scattered current noise and correlation have been shown to exhibit possible signatures of an entangled electron spin singlet state in semiconductors $\underline{25}$ This detection proposal is still far from a Bell-type measurement, but it is a necessary first step as it deals with correlations between electrons that have been extracted from their entangler and are already spatially separated.

In this paper, we first discuss in Section $\amalg$ and $\amalg$ how a double quantum dot might be used to generate pairs of entangled electrons and what problems may affect the performance of a fixed barrier double dot system as a spin entangler. Then in Section IV we introduce the dynamic turnstile configuration in order to create a regulated source of entangled electrons, and in Section $\nabla$ we discuss how the wavepacket nature of the electrons reduces the signature of electron entanglement in a beam splitter. One key aspect of our work is to analyze the proposed noise/correlation experiment of Ref. 25 in terms of particle-like wavepacket electronic states. The other key aspect is to show that it will be essentially impossible to satisfy all the stringent temporal restrictions (e.g. tunneling rates for the electrons to go in and out of the dots, the decoherence rate, etc) needed for the success of the proposed detection scheme using a static barrier double quantum dot system - we therefore suggest and analyze a situation where the double dot input/output barriers will operate in a dynamical time-dependent turnstile mode, first allowing entanglement to occur in the double dot through the exchange interaction and then enabling the extraction of the entangled electrons and their detection through noise cross-correlation measurements in the output leads.

\section{DOUBLE DOT AS AN ENTANGLEMENT FILTER: FIXED BARRIERS}

The basic idea underlying the use of a coupled double quantum dot system as an electron spin entangler has already been introduced and discussed ${ }^{3.30 .31 .32}$ It is now well-established that an exchange-coupled double quantum dot could act as an artificial molecule where individual electrons on one dot are "entangled" with electrons on the other in molecular singlet (or triplet) states, similar to the corresponding situation in real molecules. For example, a two-electron double dot with one electron on each dot (and a suitable gate to control the exchange coupling between the two dots by electrically tuning the overlap of the electron wavefunctions in the two dots) is the artificial analog of the $\mathrm{H}_{2}$ molecules on a 10 to $100 \mathrm{~nm}$ size and 0.1 to $1 \mathrm{meV}$ energy scale (although the details are qualitatively different since the "atomic" electronic confinement in the quantum dot case is the approximately parabolic external confinement potential imposed by electrostatic gates in contrast to the Coulombic nuclear confinement potential in 


\section{Double dot entangler}

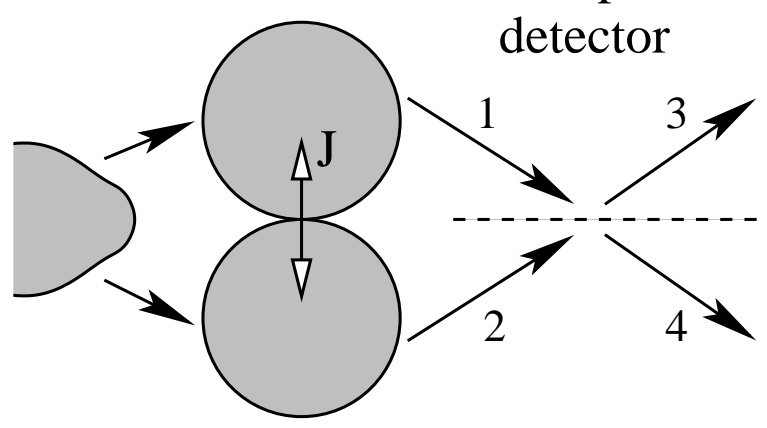

FIG. 1: Schematic diagram of a double quantum dot as an electron-spin-entanglement filter. An electron beam splitter ${ }^{51}$ is included as a potential detector for the spin entanglement ${ }^{25}$.

real atoms and molecules). In a two-electron double quantum dot the ground state (with one electron in each dot), in the absence of any external magnetic field, is the exchange-coupled and manifestly entangled spin singlet state, ${ }^{30.31}$ In multielectron (i.e. each dot containing more than one electron) double quantum dots ${ }^{32}$ the ground state, even in the absence of any applied magnetic field, could be either a singlet or a triplet or other more complicated states depending on the details of the system (e.g. the number of electrons, the confinement potential, the exchange gate behavior) just as in real many-electron molecules where the ground state may be a spin triplet state.

The idea is therefore to introduce electrons into the double dot structure through well-controlled input barrier in order for them to populate the spin singlet states and then to controllably extract the entangled electron pairs from the double dot system through output barriers into transport leads. Then the entanglement has to be detected in a suitable transport measurement which, as already proposed in Ref. 25, could be a current noise and/or cross-correlation measurement between the electrons in the entangled pair through a beam splitter (Fig. I1). The basic detection idea is simple conceptually but extremely difficult to implement experimentally. We note that the proposed current noise measurement satisfies the key criterion for establishing entanglement in the sense that these measurements involve local measurements for each constituent which are spatially separated from each other (and are no longer interacting with each other through the direct exchange coupling). Entanglement manifests itself in the correlations between local observations on spatially separated constituents. By contrast, a direct spectroscopic observation of the singlet state in the double quantum dot system itself, for example by observing the singlet-triplet energy level splitting (or the symmetric-antisymmetric gap), is in our view not direct evidence for entanglement, but evidence for the presence of inter-dot electronic coupling or wavefunction overlap. It is only by separating the two electrons, as shown schematically in Fig. [1 one could hope to demonstrate entanglement. (Demonstration of, or evidence for, entanglement necessarily requires that the local interaction originally creating the entangled state has been turned off.)

We consider a horizontally coupled double quantum dot (QD) configuration as shown in Fig. 1 where the two dots are connected to the same source electrode but different drain electrodes, so that the pair of electrons can be separated after passing through the double dot. As is evident from the schematics, such a coupled double dot has several knobs we can tune to adjust its properties because modern lithographic technologies allow various gates and leads to be put on the double dot structure in a controllable manner. These include the bias voltage applied between the source and drain electrodes, the gate voltages applied to the two quantum dots, the inter-dot tunnel coupling, and the dot-electrode couplings. Parallelly accessible double quantum dots have already been studied experimentally, ${ }_{3}^{33}$ so that the configuration discussed here should be feasible for future experimental exploration. Below we will first analyze some requirements imposed by our objective of using the double dot as an entangler for electron spins.

We assume a priori that the tunnel coupling between the double dot is stronger than the tunnel coupling between the double dot and the source and drain electrodes, so that electrons inside the double dot can form a molecular state. This is an absolutely necessary condition for our proposed spin entangler to work. This assumption also dictates that only one source electrode is needed because the electrons in the reservoir(s) would see the double dot as a single (strongly tunnel-coupled) entity. For the case of weak inter-dot tunnel coupling, we can consider the limit of vanishing coupling to understand why such a situation is not conducive to our objective of creating spin-entangled electron pairs. If there is no tunneling or wavefunction overlap between the two dots, the only possible inter-dot interaction is the classical Coulomb long range coupling. The two streams of electrons passing through the two quantum dots would have no spin correlations in this situation. The currents will be correlated because of the Coulomb blockade: when 
one of the QDs is occupied, the other would tend to stay empty to minimize the total energy of the system (depending on the applied gate voltage). The situation is quite similar to what has been studied in Ref. 34, and is not useful for the purpose of entangling electron spins. (Here is in some sense a rather simple example of a coupled system "without entanglement", precisely speaking "without spin entanglement".)

For simplicity, we consider a situation where the double dot is empty initially. This condition can be achieved by tuning the gate voltages to empty the two QDs, which has been achieved experimentally in both vertical and horizontal QDs. ${ }^{35,36,37.38}$ If the QDs are not initially empty, exchange coupling can still be established. ${ }^{32}$ However, chaotic behavior sets in as the number of electrons increases, so that the exchange coupling would be very sensitive to the energy levels involved and can vary uncontrollably in a wide range. To avoid this sensitivity to the control gate voltages, we will limit ourselves to the initially empty double dot systems for our consideration. This condition dictates that the chemical potential of the drain electrodes should be slightly lower than the ground state energy levels of the two QDs. Furthermore, since exchange coupling decreases as the level offset of the two QDs increases 30 , we will assume that the two QDs are identical with the same gate voltage, so that the ground single electron energy levels of the two dots are always aligned in order to maximize the exchange coupling. The gate voltages will be fixed to streamline the pulse sequence used to manipulate this double dot device. To make the two-electron singlet state of the double dot accessible in transport, the chemical potential of the source electrode has to be higher than the singlet state energy of the double dot with two electrons (but lower than the energy level of the two-electron triplet state, as we discuss below). In short, we require the source-drain bias voltage to enable the system to satisfy the following inequalities:

$$
E(2 e, \text { triplet })-E(1 e)>\mu_{\text {source }}>E(2 e, \text { singlet })-E(1 e) \gtrsim E(1 e)>\mu_{\text {drain }},
$$

where $E(2 e)(E(1 e))$ is the lowest energy required to put two (one) electrons in the double dot (defined relative to the bottom of the quantum dot potential well). Therefore, the source-drain bias voltage should be larger than $E(2 e$, singlet) $-E(1 e)$ (which is generally larger than $E(1 e)$ because the direct Coulomb repulsion caused increase is usually larger than the exchange caused decrease in energy) if we align the bottom of the double dot with the chemical potential of the drain electrodes, so that electrons can tunnel into the two-electron singlet from the source electrode and then empty into the drain electrodes. Notice that lower bias voltages, though not useful for the purpose of creating spin entangled electrons, may also lead to physically interesting scenarios. Under a small bias, the double dot can be considered to be a large single dot. When one excess electron occupies the double dot, the two-dot charging energy would prevent another electron from entering the double dot in the low-bias situation. The system is essentially in the Coulomb blockade regime of a single QD (with a relatively small charging energy). However, since there are two drain electrodes instead of one, when the two paths recombine (for example, at a Y-junction), electron interference effects such as Aharonov-Bohm effect can be expected as long as the system size is smaller than the electron phase coherence length.

The bias voltage needs to be small so that only one electron orbital level in each quantum dot is open to accept electrons from the source and to emit electrons to the drain. If more quantum dot orbital levels are accessible to the source electrode, electrons could tunnel into triplet and excited singlet states. The occupation of these excited states would reduce the spin filtering efficiency of the double dot. For example, if a pair of electrons are in a doubly-occupied singlet state (both electrons on the same QD), they would have higher probability for entering the same drain electrode where they would not represent a pair of distinguishable entangled electrons. These two electrons would also tend to have different energies so that their control outside the double dot becomes problematic. If the two electrons are in a triplet state, they would either leave the quantum dots in a triplet state, or undergo spin flips and possibly leave the double dot in a singlet state, though this probability is only $33 \%$ after one spin flip:

$$
\begin{aligned}
& |\uparrow \uparrow\rangle \rightarrow|\uparrow \downarrow\rangle=\left(\left|T_{0}\right\rangle+|S\rangle\right) / \sqrt{2}, \\
& |\downarrow \downarrow\rangle \rightarrow|\uparrow \downarrow\rangle=\left(\left|T_{0}\right\rangle+|S\rangle\right) / \sqrt{2}, \\
& \left|T_{0}\right\rangle \rightarrow(|\uparrow \uparrow\rangle+|\downarrow \downarrow\rangle) / \sqrt{2} .
\end{aligned}
$$

Since spin flip (or relaxation) time is quite long in QDs at low temperatures (e.g. spin-flip tunneling time in the order of tens of $\mu \mathrm{s}^{39.40}$ ), one cannot count on spin relaxation to bring a pair of electrons in a triplet state into the ground singlet state within their residence time inside the QDs (which we will show below to be in the order of 100 ps). Therefore, the bias voltage applied to the double quantum dots has to be small: $V_{\text {bias }}<E(2 e$, triplet) $-E(1 e)$, where $V_{\text {bias }} \equiv \mu_{\text {source }}-\mu_{\text {drain }}$.

The strength of dot-electrode tunneling should be sufficiently weak so that second or higher order tunneling processes (cotunneling) are strongly suppressed, because these higher order processes do not obey the spin selection rule we established by properly setting the bias voltage (so that only ground two-electron singlet state can pass through the double dot). Indeed, we believe that cotunneling would make up of a part of the current noise in the two streams of 
spin-entangled electrons, complicating the observation of the entanglement induced cross-correlation and noise in the current.

The source electrode is taken to be unpolarized (in an external magnetic field this is not necessarily the case; for example, quantum Hall edge states have been used to produce spin polarized injection into quantum dots ${ }^{41}$ ), so that any arbitrarily picked two electrons should have a spin density matrix (neglecting the exchange interaction between the two electrons and all spin-flip interactions) in which the two-spin singlet state is equally probable as any of the three triplet states:

$$
\rho=\frac{1}{4}\left(|S\rangle\left\langle S|+| T_{0}\right\rangle\left\langle T_{0}|+| T_{\uparrow}\right\rangle\left\langle T_{\uparrow}|+| T_{\downarrow}\right\rangle\left\langle T_{\downarrow}\right|\right) .
$$

It is clear from this density matrix that the double QD acts here as a spin filter that selectively allows the electron spin singlet component to tunnel through. In this sense the double QD system may be considered to be a two-spin blockade

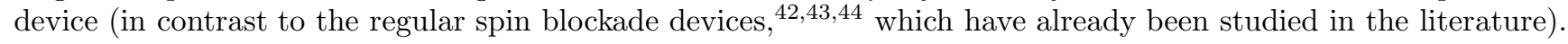

\section{PROBLEMS WITH A FIXED BARRIER DOUBLE DOT AS A SPIN ENTANGLEMENT FILTER}

When the conditions outlined in the previous section (which should be taken as the minimal necessary conditions, by no means sufficient since, for example, decoherence must be held to a minimum in the whole set-up so that entanglement can be preserved) are all satisfied, the ground two-electron singlet state becomes accessible to electrons tunneling through the double QD. However, a variety of tunneling events can occur through the double dot. It is thus crucial to analyze the relative weight of these tunneling events in order to determine whether spin entangled states would be a dominant component in the outgoing electron streams.

According to Ref. 45, the stationary current passing through a QD (treating our coupled double dot as a single QD, as illustrated in Fig. 2) can be written as

$$
I=-e \sum_{p} \sum_{\left\{n_{i}\right\}} \Gamma_{p}^{s} P\left(\left\{n_{i}\right\}\right)\left\{\delta_{n_{p}, 0} f\left(E^{i, s}(N)-E_{F}\right)-\delta_{n_{p}, 1}\left[1-f\left(E^{f, s}(N)-E_{F}\right)\right]\right\}
$$

where $V$ is the bias voltage applied across the QD, $p$ is the index of orbital energy levels inside the QD, $\left\{n_{i}\right\}$ represents dot level occupations, $\Gamma_{p}^{s(d)}$ are the tunneling matrix elements between level $p$ and the source (drain) electrode, $P\left(\left\{n_{i}\right\}\right)$ is the stationary probability for configuration $\left\{n_{i}\right\}$ given by a set of detailed balance equations, and $f$ is the FermiDirac distribution. $E^{i, s}(N)=E_{p}+U(N+1)-U(N)+\eta e V\left[E^{f, s}(N)=E_{p}+U(N)-U(N-1)+\eta e V\right]$ is the energy of the source reservoir states from (to) which an electron tunnels into (out of) state $p$ of a QD with $N$ electrons, where $E_{p}$ is the energy of a single electron state $p, U(N)$ is the Coulomb interaction energy for $N$ electrons in the QD, and $\eta e V$ is the voltage drop across the QD-source-electrode potential barrier, as shown in Fig. 2 In the simplified case we are considering here (neglecting occupation of any configuration with $N>2$ ), the only relevant electron distribution probabilities are $P(0), P(1)$, and $P(2)$ (with zero, one, and two electrons (singlet state) in the QD, and neglecting the two-electron triplet states). These three probabilities satisfy the detailed balance equations for the individual configurations $\stackrel{45}{4}$ After some algebra, we arrive at (for simplicity, assuming $T \rightarrow 0$ ) the occupation probabilities

$$
\begin{aligned}
& P(0)=\frac{1}{1+\Gamma_{1}^{s} / \Gamma_{1}^{d}+\Gamma_{1}^{s} \Gamma_{2}^{s} / \Gamma_{1}^{d} \Gamma_{2}^{d}}, \\
& P(1)=\frac{1}{1+\Gamma_{2}^{s} / \Gamma_{2}^{d}+\Gamma_{1}^{d} / \Gamma_{1}^{s}}, \\
& P(2)=\frac{1}{1+\Gamma_{2}^{d} / \Gamma_{2}^{s}+\Gamma_{1}^{d} \Gamma_{2}^{d} / \Gamma_{1}^{s} \Gamma_{2}^{s}},
\end{aligned}
$$

and a current

$$
I=e\left[\Gamma_{1}^{d} P(1)+\Gamma_{2}^{d} P(2)\right]
$$

It is clear from Eq. 4 that the probability of having two electrons in this $\mathrm{QD}$ is very sensitive to the ratio $r=\Gamma_{2}^{d} / \Gamma_{2}^{s}$. If the tunneling rate to the drain electrode $\Gamma_{2}^{d}$ is large, so that $r$ is large, $P(2)$ would be small: the QD is then almost always empty. Conversely, if the tunneling rate from the source electrode $\Gamma_{2}^{s}$ is large, so that $r$ is small, $P(2)$ would be large: the QD is almost always filled with two electrons. For our purpose of creating entanglement between electrons, which exists only in the molecular two-electron state, we need to require $r \ll 1$ (equivalently, a small $\Gamma_{2}^{d}$ ). In this case, 


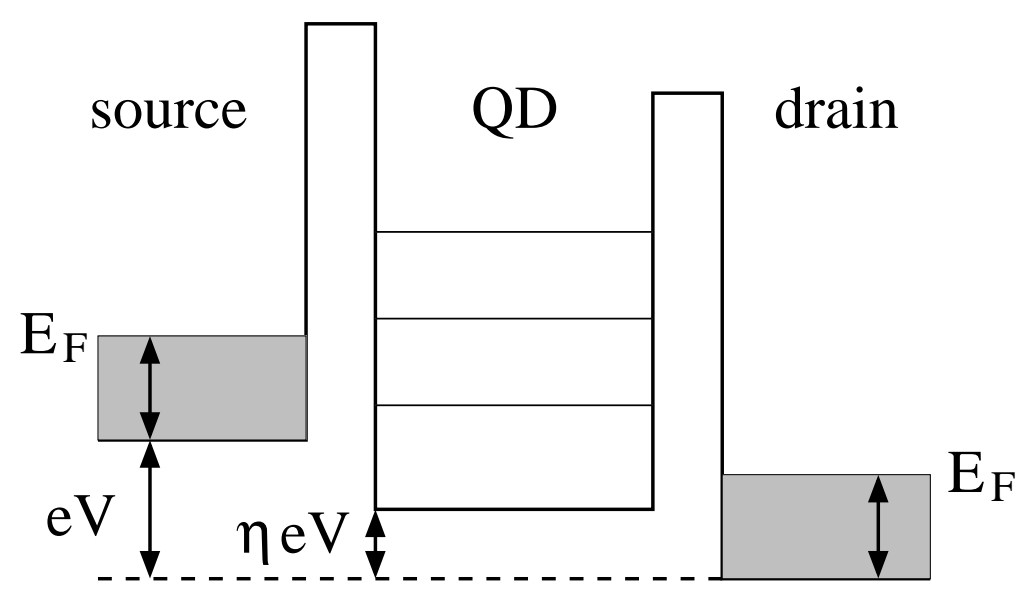

FIG. 2: Schematic diagram of a quantum dot in between a source and a drain electrode.

the current though the QD would be dominated by $\Gamma_{2}^{d} P(2)$, which corresponds to precisely one electron tunneling out of the two-electron singlet state.

To use the double dot as a source of spin-entangled electrons, the pairs of electrons have to leave the double dot with a certain degree of synchronization. However, as we have shown above, the electrical current through the double dot is dominated by single electrons tunneling out of the two-electron state. The two-electron tunneling current, as a higher order tunneling event, is proportional to $\Gamma_{2}^{d} \Gamma_{1}^{d} / U(2)$, where $U(2)$ is the two-electron Coulomb repulsion energy and is of the same order of magnitude as the single particle excitation energy and/or the external bias potential $(\mathrm{eV})$. Since we require the level broadening $\hbar \Gamma$ to be small in order to achieve state-selective tunneling, the two-electron current is much smaller than the one-electron current in our double dot configuration, especially for small $\Gamma_{2}^{d}$ and $\Gamma_{1}^{d}$.

We thus face the problem of a conflicting dichotomy in the fixed barrier double dot system in the sequential tunneling regime: it is difficult to simultaneously have a highly occupied two-electron molecular state and a high degree of synchronization for the electron pairs tunneling out of the double dot. Indeed, the output current is dominated by single electrons tunneling out of the two-electron molecular state, which would render the double dot useless as a spin entangler because the electrons coming out in transport current are entangled with electrons that are localized in the QDs and are therefore not accessible in transport measurements outside the double dot system. This problem needs to be addressed in the context of using the double dot system as a spin entangler, and this is what we do in the next Section.

\section{SYNCHRONIZATION OF OUTPUT FROM A DOUBLE DOT: A PARALLEL TURNSTILE}

It is clear from the previous sections that the key problem facing fixed barrier double quantum dot from the perspective of a spin entangler is the difficulty in controlled (regulated) extraction of pairs of entangled electrons. One way to overcome the output synchronization problem is to introduce time-dependent incoming and outgoing barriers for the double dot, particularly the outgoing barrier. This proposed introduction of time-dependent barriers for the double QD is an analog of the single electron turnstiles studied a decade ago $\underline{\underline{46}}$ Within each cycle of the turnstile, there are two stages of operation as illustrated in Fig. 3. These two stages of operations would enable us to avoid the conflicting dichotomy as each stage could optimize the individual constraints discussed in the last section. Specifically, during stage-I (Fig. Ba), the incoming barrier is low while the outgoing barrier is high, so that two electrons would occupy the double dot and form a spin singlet state. During stage-II (Fig. [3b), the incoming barrier is high and the outgoing barrier is completely removed, so that both electrons would rapidly "empty" into the two drain electrodes. The duration of the first stage is determined by the tunneling rate from the source electrode into the QDs, while the duration of the second stage would be determined by how fast the electrons diffuse out from the double dot into the drain electrodes.

To ensure synchronization for the entangled electron pairs entering the drain electrodes, the lowering of the outgoing barrier should be faster than the interdot tunneling rate. Otherwise, if one of the electrons tunnels out to the drain during the barrier lowering period, the other electron may have time to undergo charge oscillations between the two dots, therefore significantly increasing the probability that it tunnels into the same drain electrode as the other electron. Such undesirable "bunching" events, where two electrons enter the same drain electrode, would become part 


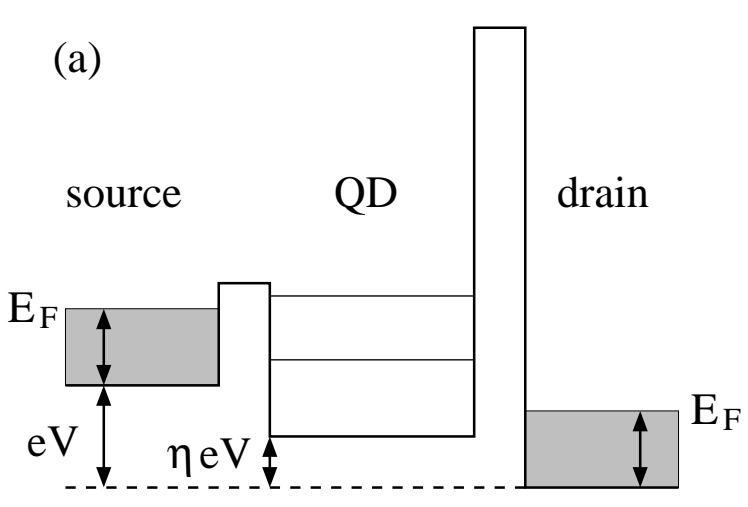

(c) incoming barrier

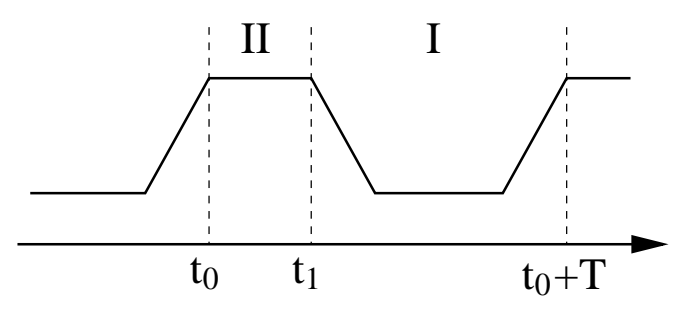

(b)

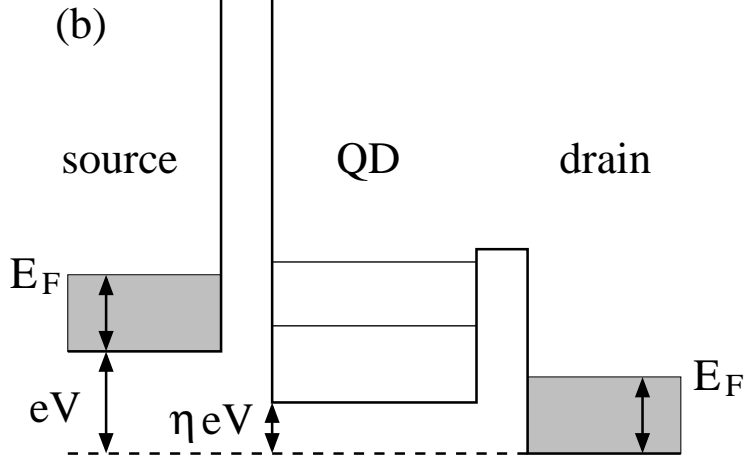

(d) outgoing barrier

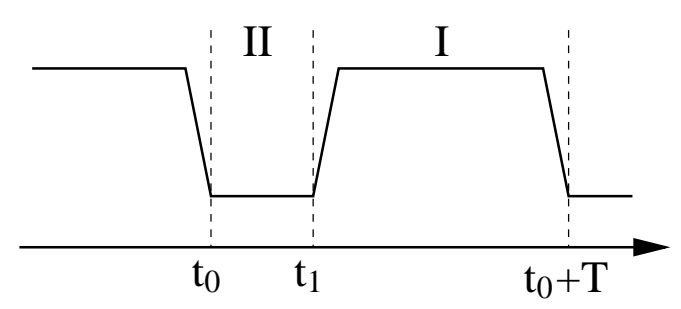

FIG. 3: The two stages of the double dot turnstile.

of the current noise in the electrical current consisting of spin-entangled electron pairs. In realistic semiconductor QDs, this condition can be barely satisfied with the best presently available microwave pulse generators. For example, if the exchange coupling between the double dot is $0.01 \mathrm{meV}(\sim 100 \mathrm{mK}$, so that the experimental temperature has to be much lower than $100 \mathrm{mK}$ in order to avoid tunneling through the two-electron triplet states), which is a reasonable number, $\stackrel{31.32}{5}$ and the on-site Coulomb repulsion energy is about $1 \mathrm{meV}$ (again a typical number), the inter-dot tunnel coupling would have to be in the order of $0.1 \mathrm{meV}$, corresponding to a tunneling time of about $40 \mathrm{ps}$. The pulse ramp up time of the present-day pulse generators is about 30 to $40 \mathrm{ps}$, comparable to the tunneling time. To improve the quality of this double dot electron spin entangler, sharper pulses are desirable.

Current up to $1 \mathrm{nA}$ is needed to evaluate current noise and cross correlation with the state-of-the-art technology. For a $1 \mathrm{nA}$ current, the electrons would stay in the QD on the average for about $160 \mathrm{ps}$. In the case of a turnstile, the corresponding repetition frequency is about $6 \mathrm{GHz}$. The inter-dot coupling can then be about $20 \mathrm{GHz}$ (tunneling time of $50 \mathrm{ps}$ ) to ensure that electrons would form molecular states inside the double dot. The barrier varying pulse width should be about $20 \mathrm{ps}$ (shorter than the inter-dot tunneling time), which is at the limit of the present technology. $\underline{47}$

An important question regarding the operation of a double dot turnstile structure as envisioned here is whether the fast gate voltage pulses would cause electron orbital or spin states to get excited. To avoid such unintended excitations, the gate voltages controlling the incoming and outgoing barriers should be varied synchronously so that the overall effect on the electrons inside the double quantum dot remains small. Adiabatic condition can then be satisfied and the electron states would remain unchanged. Quantitatively, adiabatic condition requires that $|d V / d t| / V<<J / \hbar$. The fastest rising voltage pulses have a rise time of about $30 \mathrm{ps}$, corresponding to $|d V / d t| / V \sim 30 \mathrm{GHz}$. For $J=0.1 \mathrm{meV}$, $J / \hbar \sim 100 \mathrm{GHz}$, barely satisfying the adiabatic condition above. Increasing $J$ would make the adiabatic condition easier to satisfy. Furthermore, if spin-flip interaction (such as spin-orbit coupling) is sufficiently weak in the double dot system, the only possible excitation caused by gate voltage variation would be changes in the electron orbital states. In this case the exchange $J$ in the inequality above should be replace by the single particle excitation energy $E$ that is generally at least one order of magnitude larger than $J$. Therefore, the fast voltage pulses required to operate the double dot turnstile should in general not cause serious problem in terms of exciting electrons into unwanted states. 


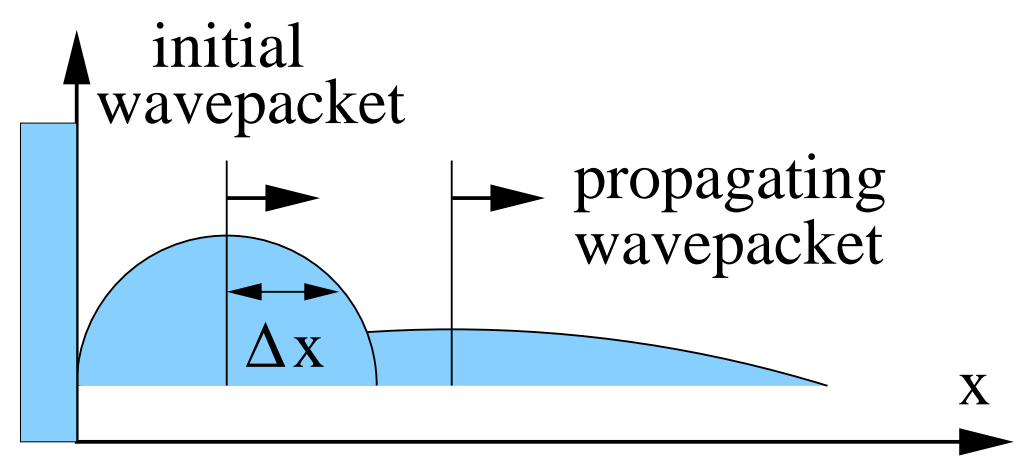

FIG. 4: Free expansion of the electron wavepackets during stage-II of the trunstile operation. The shaded area to the left of the wavepackets represents the barrier that separate the quantum dots from the source electrode. The barrier between the QDs and the drain electrodes is removed so that the QDs are now completely open, which results in the free expansion of the electron wavepackets.

\section{ELECTRON ENTANGLEMENT DETECTION USING A BEAM SPLITTER: CONSEQUENCES OF WAVEPACKET STATES}

Assuming spin-entangled electron pairs can be generated synchronously with sufficient consistency from a double dot entangler (following considerations discussed in the earlier sections), the important question arises as how to measure and quantify the degree of entanglement in the electron pairs. One approach that can take advantage of ensemble-averaging is to use a beam splitter to create two-electron interference and then measure the noise and correlation spectra of electrical currents ${ }^{25}$ Here we would like to explore whether this approach can work effectively with electron pairs coming out of the double dot entangler in the turnstile configuration discussed in the last section.

In Ref. 25, the electrons injected into the beam splitter are assumed to be in plane wave states or purely momentum states. If a double QD works as an electron spin entangler, the electrons coming out of the double dot are in expanding or dispersing wavepacket states. The use of partly localized wavepacket states instead of extended plane wave states also highlight the contrast between the quantum mechanical dual wave-particle nature of the electrons and their classical particle-like image. As shown in Fig. B] in stage-II of each turnstile cycle the outgoing barrier for the double QD is lowered to facilitate the dot-emptying process. In the simple limit that the outgoing barrier is lowered all the way so that the electron wavefunction will undergo free expansion without tunneling, as illustrated schematically in Fig. [4 the peak of the wavepacket would propagate along the drain electrode (which is assumed to be one-dimensional for simplicity) with a speed of $\pi \hbar / m a \propto \sqrt{E_{\text {conf }}}$ with $a$ being the confinement length and $E_{\text {conf }}$ the confinement energy, and the width of the wavepacket would expand with a speed $\propto \sqrt{1+\left(E_{\text {conf }} t / \hbar\right)^{2}}$ ․ㅗ For example, for a confinement length of $100 \mathrm{~nm}$, which corresponds to a confinement energy $\sim 0.5 \mathrm{meV}$, the wavepacket propagates with a speed of $1 \mu \mathrm{m}$ per $10 \mathrm{ps}$, within which period the wavepacket becomes 10 times wider. Notice that the electron dynamics here is quite different from the case of tunneling. Energy spread of an electron wavepacket would be determined by the tunneling rate $\Delta \epsilon \sim \hbar \Gamma$ if the electron tunnels through a barrier out of the quantum dot, no matter whether the system is in the turnstile or fixed barrier configuration. In our turnstile proposal here, the outgoing barrier is lowered all the way to zero during stage-II of the turnstile operation (Fig. 3i), so that the confined electron can expand out of the initial state freely into the lead without facing any barrier (Fig. [4). The energy of such an electron initially is $E=\langle H\rangle=E_{\text {conf }}$. The energy spread is determined from the initial spatial spread $\Delta x$, which is also related to the initial confinement energy $E_{\text {conf }}$ as we discussed above.

We now focus on the observability of the difference between electron singlet and triplet states assuming the electrons coming into the beam splitter (which is treated as a four-terminal device, as illustrated in Fig. II) are in wavepacket states. The current noise in each of terminals 3 and 4 and the cross correlation between currents in terminals 3 and 4 can be written as: 25.48

$$
S_{\alpha \beta}=\lim _{T \rightarrow \infty} \frac{1}{2 \pi T} \int_{0}^{T} d t e^{i \omega t}\left\langle\psi\left|\delta I_{\alpha}(t) \delta I_{\beta}(0)\right| \psi\right\rangle,
$$

where $\alpha, \beta=3,4$, and the current can be expressed in terms of the creation and annihilation operators at the terminals $: \underline{: 4}$

$$
I_{\alpha \sigma}(t)=\frac{e}{h} \sum_{E E^{\prime}}\left[a_{\alpha \sigma}^{\dagger}(E) a_{\alpha \sigma}\left(E^{\prime}\right)-b_{\alpha \sigma}^{\dagger}(E) b_{\alpha \sigma}\left(E^{\prime}\right)\right] e^{i\left(E-E^{\dagger}\right) t / \hbar}
$$


According to Ref. 25, if the electron pairs are initially in plane wave states with energies $\epsilon_{1}$ and $\epsilon_{2}$ :

$$
| \pm\rangle=\frac{1}{\sqrt{2}}\left[a_{2 \downarrow}^{\dagger}\left(\epsilon_{2}\right) a_{1 \uparrow}^{\dagger}\left(\epsilon_{1}\right) \pm a_{2 \uparrow}^{\dagger}\left(\epsilon_{2}\right) a_{1 \downarrow}^{\dagger}\left(\epsilon_{1}\right)\right]|0\rangle,
$$

the outgoing current noise and cross correlation from the beam splitter in leads 3 and 4 satisfies 25

$$
S_{\alpha \beta} \propto T(1-T)\left(1 \mp \delta_{\epsilon_{1}, \epsilon_{2}}\right),
$$

depending on whether the initial spin state is one of the triplet states or the singlet state. The physical picture of this difference is conceptually simple to understand. The two-electron singlet entangled state is antisymmetric in spin space and therefore symmetric in spatial coordinates between the two electrons (in order to maintain the overall antisymmetric nature of the two-electron fermionic state). This symmetric real space nature of the orbital two-electron wavefunction of the singlet pair then introduces the differences in the cross-correlations and the current noise spectra as the entangled electrons scattering off the beam splitter tend to "bunch" together. The observation of the noise and cross-correlation in the output currents of the beam splitter can thus be construed as a direct (transport) evidence for spin entanglement created back in the double quantum dot. Here we comment that such an interference type experiment is a solid state analog of the famous Hanbury Brown and Twiss experiment for photons ${ }^{50}$ and a natural extension of similar solid state experiments 51.52 .53 .54 with entangled electrons.

It is clear that the $\delta$-function in Eq. 9 is a direct consequence of the choice of the initial plane wave states for the electrons. A more realistic choice in our case, where electrons expand out of the quantum dots during stage-II of the turnstile cycles (see Fig. 3), would be the wavepacket states. If the orbital part of the initial wavepacket takes a simple Gaussian form:

$$
\Psi(x, t)=\frac{1}{(2 \pi)^{1 / 4} \sqrt{\Delta x}} e^{-\frac{(x-\langle x\rangle)^{2}}{4(\Delta x)^{2}}+i \frac{\langle p\rangle}{\hbar} x},
$$

where $\Delta x$ is the wavepacket width, and the average momentum $\langle p\rangle$ of the wavepacket is determined by the electron energy $E$ inside the QD and the Fermi energy in the drain electrode. The initial electronic state entering the beam splitter would now take on a more complicated form:

$$
\begin{aligned}
| \pm\rangle & =\int_{0}^{\infty} d k_{1} \int_{0}^{\infty} d k_{2} \phi_{1}\left(k_{1}\right) \phi_{2}\left(k_{2}\right) \times \frac{1}{\sqrt{2}}\left(a_{2 \downarrow}^{\dagger}\left(k_{2}\right) a_{1 \uparrow}^{\dagger}\left(k_{1}\right) \pm a_{2 \uparrow}^{\dagger}\left(k_{2}\right) a_{1 \downarrow}^{\dagger}\left(k_{1}\right)\right)|0\rangle \\
& =\int_{0}^{\infty} d \epsilon_{1} \int_{0}^{\infty} d \epsilon_{2} \psi_{1}\left(\epsilon_{1}\right) \psi_{2}\left(\epsilon_{2}\right) \times \frac{1}{\sqrt{2}}\left(a_{2 \downarrow}^{\dagger}\left(\epsilon_{2}\right) a_{1 \uparrow}^{\dagger}\left(\epsilon_{1}\right) \pm a_{2 \uparrow}^{\dagger}\left(\epsilon_{2}\right) a_{1 \downarrow}^{\dagger}\left(\epsilon_{1}\right)\right)|0\rangle,
\end{aligned}
$$

where

$$
\phi_{i}(k)=\frac{2}{(2 \pi)^{1 / 4}} \sqrt{\frac{\Delta x}{L}} e^{-\left(k-k_{i}\right)^{2}(\Delta x)^{2}} e^{i \theta_{i}}
$$

Here $\theta_{i}=E_{k} t_{i} / \hbar$ is an initial phase determined by when the wavepacket enters the lead. Notice that there is no time-dependence in this wavepacket wavefunction because we are working in the Heisenberg picture (see Eq. (77)). Physically, the wavepacket state is made up of a continuous spectrum of plane wave states, and represents a spatially localized electron. Going through similar type of algebra as in Ref. 25 using wavepacket states rather than plane wave states, we arrive at the expression for the current noise and cross correlation:

$$
S_{\alpha \beta} \propto T(1-T)\left(1 \mp\left|\int d k \phi_{1}^{*}(k) \phi_{2}(k)\right|^{2}\right) .
$$

Thus, in the case of a simple Gaussian wavepacket, the noise/cross-correlation function is given by (with $\alpha$ and $\beta$ being 3 or 4 ),

$$
S_{\alpha \beta} \propto T(1-T)\left[1 \mp \frac{1}{1+d^{2} / 4} e^{-\frac{1}{1+d^{2} / 4}\left(k_{1}-k_{2}\right)^{2}(\Delta x)^{2}} e^{-\frac{d^{2}}{2+d^{2} / 2}\left(k_{1}^{2}+k_{2}^{2}\right)(\Delta x)^{2}}\right]
$$

where $d=\hbar\left(t_{1}-t_{2}\right) / 2 m(\Delta x)^{2}$ represents the difference between the initial phases of the two wavepackets (when they each entered the leads), and $k_{1} \sim\langle p\rangle_{1} \propto \sqrt{\epsilon_{1}}$ and $k_{2} \sim\langle p\rangle_{2} \propto \sqrt{\epsilon_{2}}$. The strength of the signature of electron spin singlet/triplet states is thus determined by the spectral overlap of the two electron wavepackets coming into 
the beam splitter relative to the spectral width of each individual wavepacket, and when they each enter the leads (therefore the beam splitter). In the double dot turnstile configuration, $\epsilon_{i}$ 's are determined by the energy levels inside the dots (assuming the two drain electrodes have the same Fermi energy). The energy levels of the two quantum dots need to be aligned not only to maximize the interdot exchange coupling $J$, but also keep $k_{1}-k_{2}$ to be relatively small. $\Delta x$ takes on the value of confinement length in the case of a turnstile when the outgoing barrier is completely removed during stage-II in Fig. (3). It can be estimated that for reasonable initial energy differences the $\left[\left(k_{1}-k_{2}\right) \Delta x\right]^{2}$ factor in Eq. (14) should be smaller than one so that the first exponential factor in Eq. (14) should not suppress the interference signal by much. If the electrons enter the beam splitter (thus the drain electrodes) at the same time, so that $d \propto t_{1}-t_{2}=0$, the expression in Eq. 14 can be simplified:

$$
S_{\alpha \beta} \propto T(1-T)\left[1 \mp e^{-\left(k_{1}-k_{2}\right)^{2}(\Delta x)^{2}}\right]
$$

In this case the contrast between entangled (singlet) and non-entangled electron pairs should be mostly preserved, so that plain wave description of the electrons ${ }^{25}$ should work reasonably well. If the electrons do not enter the beam splitter simultaneously due to reasons such as differences in the two outgoing barriers or difference in the lengths of the electrodes between the QDs and the beam splitter, the effect of the initial phase difference is non-negligible. For example, for $\Delta x \sim 10 \mathrm{~nm}, d \sim 10^{13}\left(t_{1}-t_{2}\right)$. If $t_{1}-t_{2}$ is in the order of 10 picosecond, which could be the case if the outgoing barrier of the double dot turnstile is modulated on the time scale of 30 picosecond, the factor $d$ satisfies $d \gg 1$. This re-enforces the fact that the first exponential factor in Eq. (14) is not very important in the present case (because of the $1 /\left(1+d^{2} / 4\right)$ factor in the exponent). The exponent of the second exponential factor can be approximated by $2\left(k_{1}^{2}+k_{2}^{2}\right) \Delta x^{2}$, which is in the order of 1 for $\Delta x \sim 10 \mathrm{~nm}$ and the average electron energy $\sim 1$ meV. If electrons have larger kinetic energy, the difference between singlet and triplet states would be significantly suppressed in the signal from the beam splitter.

When the electrons come from a fixed barrier double dot, the spectral width $\Delta \epsilon$ for each wavepacket is determined by the decay rate through the outgoing potential barrier, which is much smaller than the confinement energy $\left(\hbar \Gamma \ll E_{\text {conf }}\right.$ so that bound states in the QDs are well defined). Now $\Delta x$ would generally be very large (for a dot lifetime of 1 ns, $\Delta x$ could be larger than $10 \mu \mathrm{m})$, so that $d=\hbar\left(t_{1}-t_{2}\right) / 2 m(\Delta x)^{2}$ is small even for $t_{1}-t_{2}$ in the order of ns (here we neglect effects from electrons not entangled, which would themselves cause deterioration in the signal). Now the first exponential factor in Eq. (14) would dominate, so that even a small energy difference of 0.01 meV can cause significant signal suppression. This situation is a direct extension of treating electrons as plane waves, for which the spectral width $\Delta \epsilon$ goes to zero. The result here shows again the advantage of the double QD turnstile configuration as compared to the fixed barrier QDs from the perspective of the signal to noise ratio in this particular beam splitter detection scheme.

If the beam splitter is too far downstream from the double dot entangler, the wavepackets would lose their phase coherence through scattering, and the two-electron spin state deteriorates through spin decoherence and relaxation, so that the strength of the signature of singlet/triplet states would reduce accordingly ${ }^{55}$

\section{DISCUSSION}

With a double dot turnstile, only the singlet spin-entangled state $|\uparrow \downarrow\rangle-\downarrow \uparrow\rangle$ can be filtered from an unpolarized reservoir. Although an external magnetic field can make the triplet state the ground state of a double dot,30.31.32 the lowest energy state of the triplet would be a polarized unentangled state (e.g. $|\uparrow \uparrow\rangle)$. To generate the other three Bell states (maximally entangled two-spin states: $|\uparrow \downarrow\rangle+\downarrow \uparrow\rangle$ and $|\uparrow \uparrow\rangle \pm|\downarrow \downarrow\rangle)$, the double dot turnstile must be combined with single spin operations such as phase shift and spin flip.

One potential problem with the turnstile configuration is the unintended inter-gate cross talk because of the simultaneous presence of closely-packed gates (needed to produce small quantum dots so that the exchange coupling between the two dots is sizable) and the relatively high operational frequency for the turnstile (6 GHz). From a control point of view, the residence time for the electrons inside the quantum dots should be as long as possible $(>1$ ns) in order to lower the operational frequency of the turnstile. However, the corresponding smaller current would lead to a suppression of the signal-to-noise ratio in the final measurement, particularly if the measurement device is a beam splitter, since current noise and correlation measurement require a relatively large current (in the order of $\mathrm{nA}$ ). Future development in the current measurement techniques would certainly help the experimental demonstration of the double dot turnstile entangler through the beam splitter detection scheme.

Another question regarding the turnstile scheme is whether rapidly varying potential barriers would significantly increase the energy discrepancy between the two electrons as they leave the quantum dot for the leads. A simple estimate can help clarify this issue. The fastest pulse rise/fall time available now is about 30 ps. Using the uncertainty principle, the corresponding energy uncertainty is smaller than $0.1 \mathrm{meV}$. Recall that $\Delta x$ is in the orders of $10 \sim 100$ 
$\mathrm{nm}$, the exponent $(\Delta k \Delta x)^{2}$ in Eq. 14 can be estimated to be in the order of $10^{-2} \sim 10^{-4}$ if $E_{\text {conf }}$ is $\sim 1 \mathrm{meV}$. Similarly, the added fluctuation in energy would not cause significant increase in $\left(k_{1}^{2}+k_{2}^{2}\right) \Delta x^{2}$ either because the wavepacket energies $\epsilon_{i}=\hbar^{2} k_{i}^{2} / 2 m$ are already in the order of meV to begin with. Therefore, the energy fluctuations caused by the fast pulses operating the turnstile should not lead to any significant suppression of the current noise and correlation signal after the beam splitter.

In this paper we studied how the efficiency of a beam splitter is reduced because of the wavepacket nature of the conduction electrons. There are also other physical processes that lead to decreased sensitivity for a beam splitter ${ }^{55.56}$ For example, it has been shown that the presence of spin-orbit coupling in the vicinity of a beam splitter, which is particularly true for semiconductor nanostructures based on two-dimensional electron gas (2DEG), can hurt the detection efficiency of a beam splitter ${ }^{56}$ Indeed, the asymmetric nature of the 2DEG and the associated spin-orbit coupling pose a potential problem to the general study of spin-based quantum computing near interfaces and need to be further studied.

The most important issue in the observability of electronic spin entanglement in this context is perhaps the problem of decoherence, which is usually severe for electrons in solid state systems. The question is the extent to which spin entanglement, even if it is successfully produced in the double dot spin entangler, will survive through the outgoing leads to be detected in the current noise correlation measurements. One would want to have the temperature as low as possible and the transport should be ballistic through the leads in order to minimize phase breaking scattering. Ideally one desires current leads which are devoid of electrons themselves (e.g. carbon nanotubes ${ }^{21}$ ), but are capable of conducting current so as to eliminate electron-electron interaction effects as much as possible. In reality, the leads are likely to be metallic, and one must make them reasonably short in order not to lose entanglement as the electrons transverse through the leads ${ }^{25}$ Whether this can be achieved or not experimentally is unknown at this stage.

\section{CONCLUSION}

In this paper we study the conditions necessary for a double quantum dot system to work as an efficient electron spin entangler. We have analyzed in detail the required relative strength of the tunneling matrix elements, and the desirable bias and gate voltages. The difficulties with fixed barrier double dots, especially with extracting electron pairs, are carefully clarified, thus establishing the necessity of time-dependent input/output barriers which would enable optimization of the various tunneling rates needed for producing entanglement and for regularly exporting pairs of spin-entangled electrons. We discuss the conditions on the turnstile configuration for the double dot to be an efficient generator of spin-entangled electron pairs. We also analyze the consequences of wavepacket states for the electrons as they leave the double dot. We show that a double quantum dot turnstile is, in principle, an efficient electron spin entangler or entanglement filter because of the exchange coupling between the dots and the controllable electron output that is possible in such a device. Whether electronic entanglement in a double quantum dot system can be experimentally detected in a transport noise/correlation measurement would depend on many conditions, including the coherence in the output leads, but our work shows that, as a matter of principle, such transport measurements are feasible.

We thank LPS, ARDA, and ARO for partial financial support. We also thank useful discussions with Y.Z. Chen and Richard Webb.

1 M.A. Nielsen and I.L. Chuang, Quantum computation and quantum information (Cambridge, New York, 2000).

2 C.H. Bennett and D.P. DiVincenzo, Nature 404, 247 (2000).

3 D. Loss and D.P. DiVincenzo, Phys. Rev. A 57, 120 (1998).

${ }^{4}$ R. Vrijen, E. Yablonovitch, K. Wang, H.W. Jiang, A. Balandin, V. Roychowdhury, Tal Mor, and D.P. DiVincenzo, Phys. Rev. A 62, 012306 (2000).

5 B.E. Kane, Nature (London) 393, 133 (1998).

${ }^{6}$ V. Privman, I.D. Vagner, and G. Kventsel, Phys. Lett. A 239, 141 (1998); D. Mozyrsky, V. Privman, and M.L. Glasser, Phys. Rev. Lett. 86, 5112 (2001).

7 A.J. Skinner, M.E. Davenport, and B.E. Kane, Phys. Rev. Lett. 90, 087901 (2003).

8 A. Aspect, Nature 398, 189 (1999).

${ }^{9}$ W. Tittel, J. Brendel, H. Zbinden, and N. Gisin, Phys. Rev. Lett. 81, 3563 (1998).

10 G. Weihs, T. Jennewein, C. Simon, H. Weinfurter, and A. Zeilinger, Phys. Rev. Lett. 81, 5039 (1998).

11 M.A. Rowe, D. Kielpinski, V. Meyer, C.A. Sackett, W.M. Itano, C. Monroe, and D.J. Wineland, Nature 409, 791 (2001).

12 The mean field Hartree-Fock Slater determinant states of electrons in solids (or atoms or molecules) cannot be factored into a product of single particle states because they incorporate Pauli principle through the appropriate antisymmetrization 
(thus reflect the fermionic characteristics of electrons). However, such correlation is not nonlocal. When the particles are separated spatially, the antisymmetrization does not carry physical meaning anymore. Therefore, single Slater determinant states should not be considered entangled. ${ }^{13}$

13 J. Schliemann, J.I. Cirac, M. Kus, M. Lewenstein, and D. Loss, Phys. Rev. A 64, 022303 (2001).

14 If two entangled objects weakly interacts with each other, but rapid measurement is available, Bell-type comparison of measurement results may still be possible with high fidelity ${ }^{11}$ However, the measurement techniques (for spins, for example) are generally quite slow in solids so that such scenario is generally difficult to realize.

15 Observation of entanglement requires that the interaction that creates the entangled state be turned off when the nonlocal correlations are measured between the entangled pairs - there is nothing unusual about finding correlations between objects that are strongly interacting with each other. For this very same reason interaction or coupling induced mode repulsion or resonant level splitting, which is fairly common in solid state spectroscopic measurements, is not direct evidence for entanglement - such resonant level repulsion is only evidence for coupling between two objects. (In fact, such level repulsion by itself is not even evidence for a quantum mechanical coupling between the two systems or energy levels since two coupled classical oscillators give rise to qualitatively identical mode repulsion or level splitting behavior.)

16 S. Das Sarma, J. Fabian, X. Hu, and I. Zutic, cond-mat/0002256 IEEE Trans. Magn. 36, 2821 (2000).

17 G.B. Lesovik, T. Martin, and G. Blatter, Euro. Phys. J. B 24, 287 (2001).

18 N.M. Chtchelkatchev, G. Blatter, G.B. Lesovik, and T. Martin, Phys. Rev. B 66, 161320 (2002).

19 P. Recher, E.V. Sukhorukov, and D. Loss, Phys. Rev. B 63, 165314 (2001).

20 P. Recher and D. Loss, Phys. Rev. B 65, 165327 (2002).

21 C. Bena, S. Vishveshwara, L. Balents, and M.P.A. Fisher, Phys. Rev. Lett. 89, 037901 (2002).

${ }^{22}$ R. Ionicioiu, P. Zanardi, F. Rossi, Phys. Rev. A 63, 050101 (2001).

${ }^{23}$ W. D. Oliver, F. Yamaguchi, and Y. Yamamoto, Phys. Rev. Lett. 88, 037901 (2002).

24 D.S. Saraga and D. Loss, Phys. Rev. Lett. 90, 166803 (2003).

25 G. Burkard, D. Loss, and E.V. Sukhorukov, Phys. Rev. B 61, R16303 (2000).

26 A.T. Costa and S. Bose, Phys. Rev. Lett. 87, 277901 (2001).

27 S. Bose and D. Home, Phys. Rev. Lett. 88, 050401 (2002).

28 P. Samuelsson, E.V. Sukhorukov, and M. Buttiker, Phys. Rev. Lett. 91, 157002 (2003).

${ }^{29}$ C.W.J. Beenakker, C. Emary, M. Kindermann, and J.L. van Velsen, cond-mat/0305110

30 G. Burkard, D. Loss, and D.P. DiVincenzo, Phys. Rev. B 59, 2070 (1999).

31 X. Hu and S. Das Sarma, Phys. Rev. A 61, 062301 (2000).

32 X. Hu and S. Das Sarma, Phys. Rev. A 64, 042312 (2001).

33 A.W. Holleitner, R.H. Blick, and K. Eberl, Appl. Phys. Lett. 82, 1887 (2003); Y.Z. Chen and R.A. Webb, unpublished.

${ }^{34}$ F. Hofmann, T. Heinzel, D.A. Wharam, J.P. Kotthaus, G. Böhm, W. Klein, G. Tränkle, and G. Weimann, Phys. Rev. B 51, 13872 (1995); F. Hofmann and D.A. Wharam, Advances in Solid State Phys. 35, 197-213 (1996).

35 L.P. Kouwenhoven, D.G. Austing, and S. Tarucha, Rep. Prog. Phys. 64, 701 (2001).

${ }^{36}$ W.G. van der Wiel, S. De Franceschi, J.M. Elzerman, T. Fujisawa, S. Tarucha, and L.P. Kouwenhoven LP, Rev. Mod. Phys. 75, 1 (2003).

37 M. Ciorga, A.S. Sachrajda, P. Hawrylak, C. Gould, P. Zawadzki, S. Jullian, Y. Feng, and Z. Wasilewski, Phys. Rev. B 61, R16315 (2000).

38 J.M. Elzerman, R. Hanson, J.S. Greidanus, L.H. Willems van Beveren, S. De Franceschi, L.M.K. Vandersypen, S. Tarucha, and L.P. Kouwenhoven, cond-mat/0212489

39 T. Fujisawa, D.G. Austin, Y. Tokura, Y. Hirayama, and S. Tarucha, Nature 419, 278 (2002).

40 R. Hanson, B. Witkamp, L.M.K. Vandersypen, L.H. Willems van Beveren, J.M. Elzerman, and L.P. Kouwenhoven, cond-mat/0303139

41 A.S. Sachrajda, P. Hawrylak, M. Ciorga, C. Gould, and P. Zawadzki, Physica E 10, 493 (2001).

42 K. Ono, D.G. Austin, Y. Tokura, and S. Tarucha, Science 297, 1313 (2002).

43 A.K. Hüttel, H. Qin, A.W. Holleitner, R.H. Blick, K. Neumaier, D. Weinmann, K. Eberl, and J.P. Kotthaus, cond-mat/0109104

44 M. Ciorga, A. Wensauer, M. Pioro-Ladriere, M. Korkusinski, J. Kyriakidis, A.S. Sachrajda, and P. Hawrylak, Phys. Rev. Lett. 88, 256804 (2002).

45 C.W.J. Beenakker, Phys. Rev. B 44, 1646 (1991).

46 L.P. Kouwenhoven, A.T. Johnson, N.C. van der Vaart, C.J.P.M. Harmans, and C.T. Foxon, Phys. Rev. Lett. 67, 1626 (1991); L.P. Kouwenhoven, A.T. Johnson, N.C. van der Vaart, A. van den Enden, C.J.P.M. Harmans, and C.T. Foxon, Z. Phys. B 85, 381 (1991).

47 T. Fujisawa, Y. Tokura, and Y. Hirayama, Phys. Rev. B 63, 081304(R) (2001).

48 M. Buttiker, Phys. Rev. Lett. 65, 2901 (1990); Phys. Rev. B 46, 12485 (1992).

49 L.I. Schiff, Quantum Mechanics (McGraw-Hill, New York, 1968).

50 R. Hanbury Brown and R.Q. Twiss, Nature 178, 1447 (1956).

51 R.C. Liu, B. Odom, Y. Yamamoto, and S. Tarucha, Nature 391, 263 (1998).

52 W.D. Oliver, J. Kim, R.C. Liu, and Y. Yamamoto, Science 284, 299 (1999); M. Henny, S. Oberholzer, C. Strunk, T. Heinzel, K. Ensslin, M. Holland, and C. Schönenberger, ibid. 284, 296 (1999).

53 X. Maitre, W.D. Oliver, Y. Yamamoto, Physica E 6, 301 (2000).

${ }^{54}$ Y. Ji, Y.C. Chung, D. Sprinzak, M. Heiblum, D. Mahalu, and H. Shtrikman, Nature 422, 415 (2003).

55 G. Burkard and D. Loss, Phys. Rev. Lett. 91, 087903 (2003). 
56 J.C. Egues, G. Burkard, and D. Loss, Phys. Rev. Lett. 89, 176401 (2002). 